\title{
The CAD Modelling Possibilities of Gear Pairs by Two Ways in the Mechanical Engineering Practice
}

\author{
S. BoDZÁS ${ }^{1}$, Zs. BÉKÉSI ${ }^{2}$, J. KERTÉSZ ${ }^{3}$, T. SZORCSIK ${ }^{4}$ \\ 1University of Debrecen, Faculty of Engineering, Department of Mechanical Engineering, \\ bodzassandor@eng.unideb.hu \\ ${ }^{2}$ University of Debrecen, Faculty of Engineering, Department of Air- and Load Vehicle, zsolt.bekesi@eng.unideb.hu \\ ${ }^{3}$ University of Debrecen, Faculty of Engineering, Department of Air- and Load Vehicle, \\ kertesz.jozsef@eng.unideb.hu \\ 4TRIGON Electronica Ltd., tamas.szorcsik@trigone.hu
}

\begin{abstract}
The geometric design of the gear pairs can happen by unique programs or the GearTeq 2021 software. The second one is a complex gear designer software which is capable to design different types of gears based on the initial geometric parameters. Naturally, the users' competence for the gear theorem and the manufacturing technology is necessary for the design process. Gear design and modelling processes are shown in this publication. The designed gear pairs are usable in different engineering fields (robots, working machines, vehicles, etc.).
\end{abstract}

Keywords: GearTeq 2021, gears, design, CAD, model

\section{Introduction}

The aim of the study is the geometric modelling by computer way because of the further tooth contact analysis (TCA) and manufacturing design.

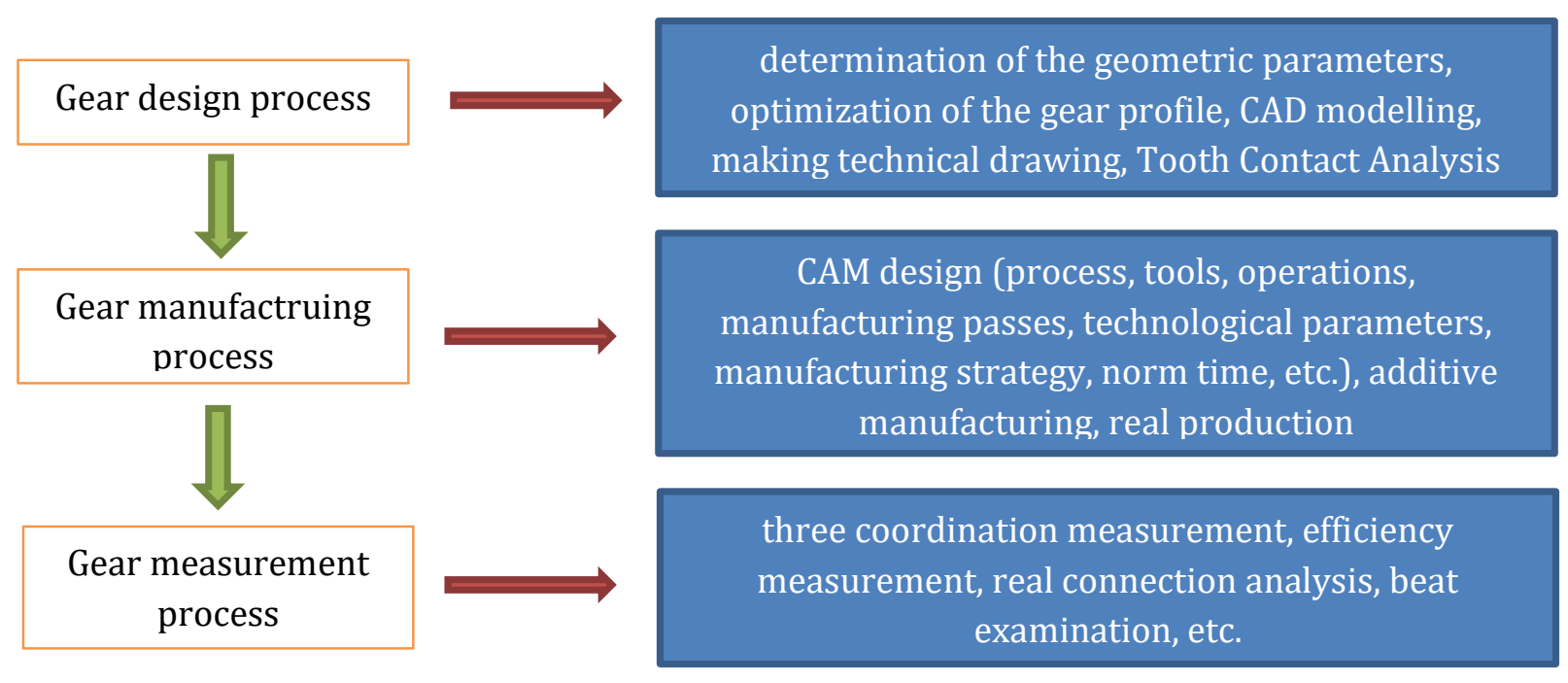

Figure 1. The complex gear generation process 
The main steps of the gear generation process can be seen on Figure 1. Originally, three main fields can be created: design, manufacturing and measurement. The aim of the TCA is to analyse the mechanical parameters of the connecting gear pairs for different loads and the optimization of the gear geometry considering the mechanical results.

\section{Gear design and modelling by Matlab and SolidWorks software}

In case of special gears which have unique geometry making a mathematical software is reasonable to ease the calculation process and to determine to gear profile. The requirements of this software are the calculation of the geometric parameters based on the references [3-11] and generation of the gear profile $[1-4,6,8,10]$ for the CAD software. Similar unique software was written for spur gears and helical gears (having modified teeth, normal teeth and x-zero type) and bevel gears having straight teeth. After the geometric design and profile drawing by Matlab software the profile points can be saved into txt format for the CAD software (SolidWorks). Interpolation B-spline curve can be inserted for the profile points in the SolidWorks. Considering the calculated geometric parameters the CAD models can be designed. Some existing results can be seen on Figure 2 and 3.
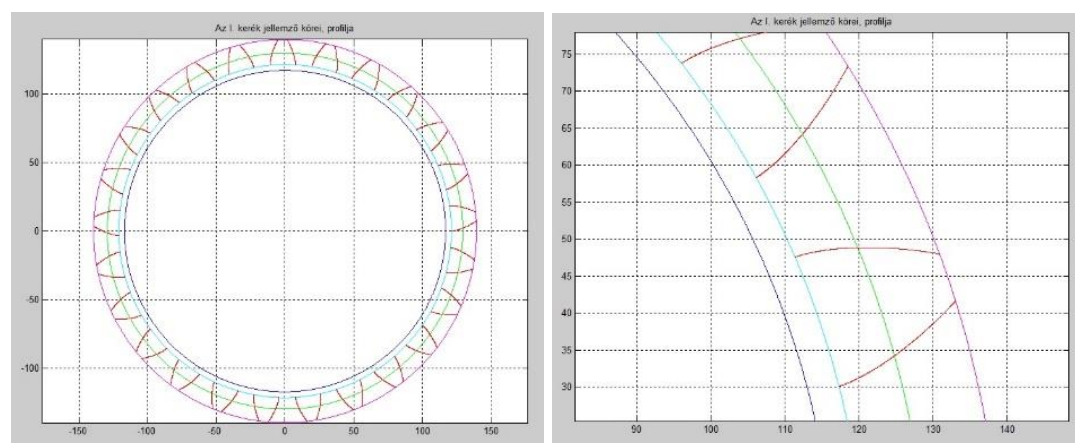

a) pinion
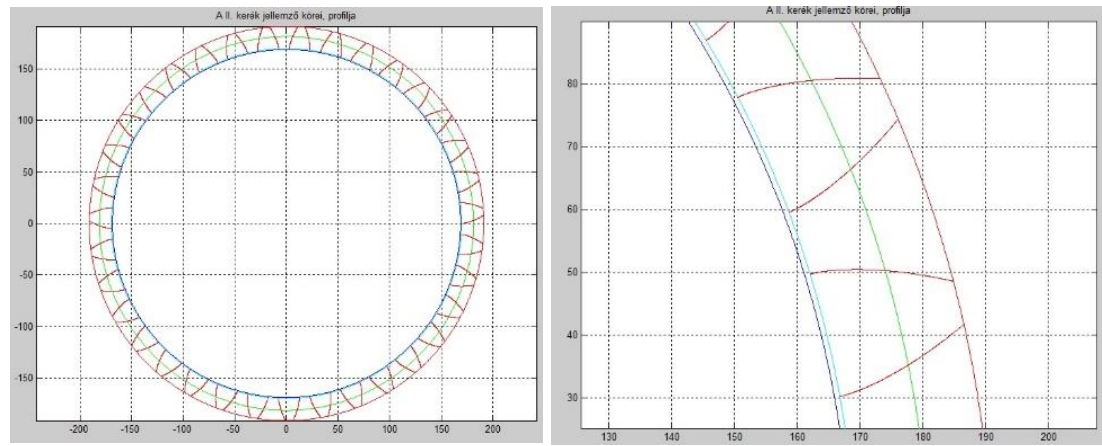

b) gear

\begin{tabular}{|l|c|c|}
\hline X-zero type helical gear pair & Pinion & Gear \\
\hline Axial module & \multicolumn{2}{|c|}{$\mathrm{m}=10 \mathrm{~mm}$} \\
\hline Number of teeth & $\mathrm{z}_{1}=25 \quad \mathrm{z}_{2}=35$ \\
\hline Helix angle & \multicolumn{2}{|c|}{$\beta_{0}=15^{\circ}$} \\
\hline Lead angle & \multicolumn{2}{|c|}{$\gamma_{0}=75,923^{\circ}$} \\
\hline $\begin{array}{l}\text { Involute profile angle on the } \\
\text { pitch circle in axial section }\end{array}$ & \multicolumn{2}{|c|}{$\alpha_{0 \mathrm{~h}}=20,646^{\circ}$} \\
\hline
\end{tabular}




\begin{tabular}{|c|c|c|}
\hline Thread pitch in axial section & \multicolumn{2}{|c|}{$\mathrm{t}_{\mathrm{oh}}=32,524 \mathrm{~mm}$} \\
\hline Addendum & \multicolumn{2}{|c|}{$\mathrm{h}_{\mathrm{a}}=10 \mathrm{~mm}$} \\
\hline Clearence & \multicolumn{2}{|c|}{$\mathrm{c}=2,5 \mathrm{~mm}$} \\
\hline Dedendum & \multicolumn{2}{|c|}{$\mathrm{h}_{\mathrm{f}}=12,5 \mathrm{~mm}$} \\
\hline Elementary centre distance & \multicolumn{2}{|c|}{$\mathrm{a}_{0}=310,582 \mathrm{~mm}$} \\
\hline Pitch circle diameters & $\mathrm{d}_{1}=258,819 \mathrm{~mm}$ & $\mathrm{~d}_{2}=362,346 \mathrm{~mm}$ \\
\hline Outside diameters & $\mathrm{d}_{\mathrm{a} 1}=278,819 \mathrm{~mm}$ & $\mathrm{~d}_{\mathrm{a} 2}=382,346 \mathrm{~mm}$ \\
\hline Root diameters & $\mathrm{d}_{\mathrm{f} 1}=233,819 \mathrm{~mm}$ & $\mathrm{~d}_{\mathrm{f} 2}=337,346 \mathrm{~mm}$ \\
\hline Backlash & \multicolumn{2}{|c|}{$\mathrm{j}_{\mathrm{s}}=1.6262 \mathrm{~mm}$} \\
\hline Working depth & \multicolumn{2}{|c|}{$\mathrm{h}_{\mathrm{w}}=20 \mathrm{~mm}$} \\
\hline Tooth thickness & \multicolumn{2}{|c|}{$S_{a x}=15,449 \mathrm{~mm}$} \\
\hline Base diameters & $\mathrm{d}_{\mathrm{ak} 1}=242,19 \mathrm{~mm}$ & $\mathrm{~d}_{\mathrm{ak} 2}=339,07 \mathrm{~mm}$ \\
\hline Transmission ratio & \multicolumn{2}{|c|}{$\mathrm{i}=1,4$} \\
\hline
\end{tabular}

c) The calculated geometric parameters

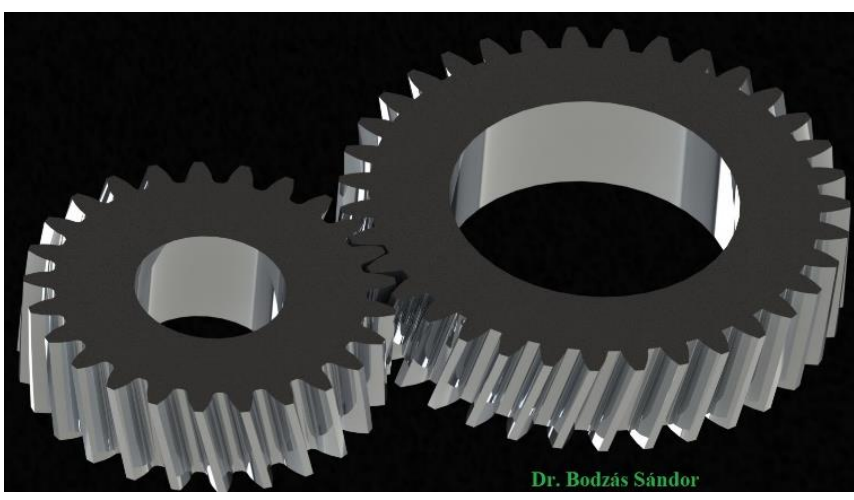

d) CAD models of the elements

Figure 2. Geometric design of an X-zero type helical gear drive [1]

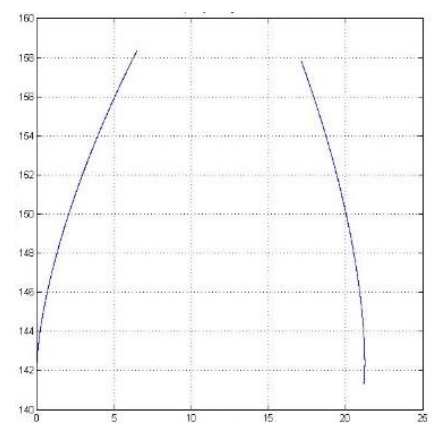

a) profile on the lowest diameter of the pinion

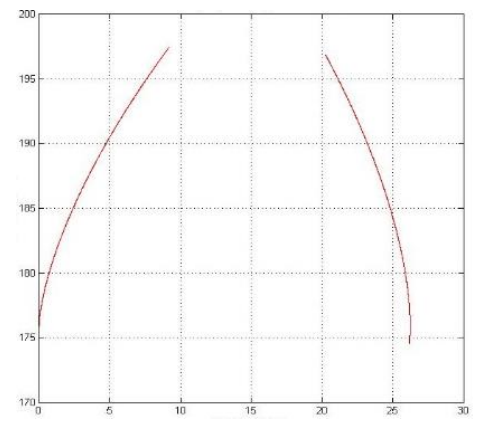

b) profile on the highest diameter of the pinion 

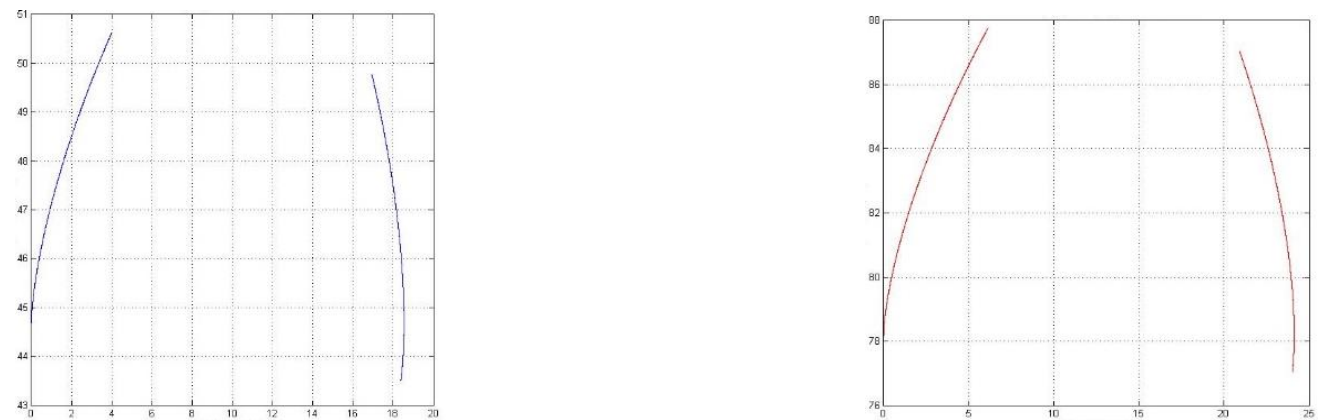

c) profile on the lowest diameter of the gear

d) profile on the highest diameter of the gear

\begin{tabular}{|c|c|c|}
\hline $\begin{array}{c}\text { The parameters of a bevel gear pair } \\
\text { having straight teeth }\end{array}$ & Pinion & Gear \\
\hline Module & \multicolumn{2}{|c|}{$\mathrm{m}=15 \mathrm{~mm}$} \\
\hline Number of teeth & $\mathrm{z}_{1}=20$ & $\mathrm{z}_{2}=30$ \\
\hline The largest pitch circle diameters & $\mathrm{d}_{01}=300 \mathrm{~mm}$ & $\mathrm{~d}_{02}=450 \mathrm{~mm}$ \\
\hline Half pitch angle of the pitch circle & $\delta_{01}=56.3^{\circ}$ & $\delta_{02}=33.69^{\circ}$ \\
\hline Effective pitch surface radius & \multicolumn{2}{|c|}{$\mathrm{R}_{\mathrm{e}}=270.416 \mathrm{~mm}$} \\
\hline Addendum on the largest diameter & \multicolumn{2}{|c|}{$\mathrm{f}_{0}=15 \mathrm{~mm}$} \\
\hline Dedendum on the largest diameter & \multicolumn{2}{|c|}{$\mathrm{l}_{0}=18 \mathrm{~mm}$} \\
\hline The largest outside diameters & $\mathrm{d}_{\mathrm{f} 1}=316.64 \mathrm{~mm}$ & $\mathrm{~d}_{\mathrm{f} 2}=474.96 \mathrm{~mm}$ \\
\hline The largest root diameters & $\mathrm{d}_{\mathrm{a} 1}=280.03 \mathrm{~mm}$ & $\mathrm{~d}_{\mathrm{a} 2}=420.04 \mathrm{~mm}$ \\
\hline Face width & \multicolumn{2}{|c|}{$\mathrm{b}=77.26 \mathrm{~mm}$} \\
\hline Dedendum angle & \multicolumn{2}{|c|}{$\lambda=3.8^{\circ}$} \\
\hline Tip cone angle & $\delta_{f 1}=60.11^{\circ}$ & $\delta_{f 2}=37.49^{\circ}$ \\
\hline Root cone angle & $\delta_{l 1}=52.5^{\circ}$ & $\delta_{l 2}=29.88^{\circ}$ \\
\hline $\begin{array}{l}\text { Circular pitch on the largest pitch circle } \\
\text { diameter }\end{array}$ & \multicolumn{2}{|c|}{$\mathrm{t}=47.123 \mathrm{~mm}$} \\
\hline Clearance at flank & \multicolumn{2}{|c|}{$\mathrm{j}_{\mathrm{s}}=2.356 \mathrm{~mm}$} \\
\hline $\begin{array}{l}\text { Pitch circle tooth thickness on the } \\
\text { largest diameters }\end{array}$ & \multicolumn{2}{|c|}{$\mathrm{S}_{\mathrm{ax}}=21.2 \mathrm{~mm}$} \\
\hline Transmission ratio & \multicolumn{2}{|c|}{$\mathrm{i}=1.5$} \\
\hline
\end{tabular}

e) The calculated geometric parameters

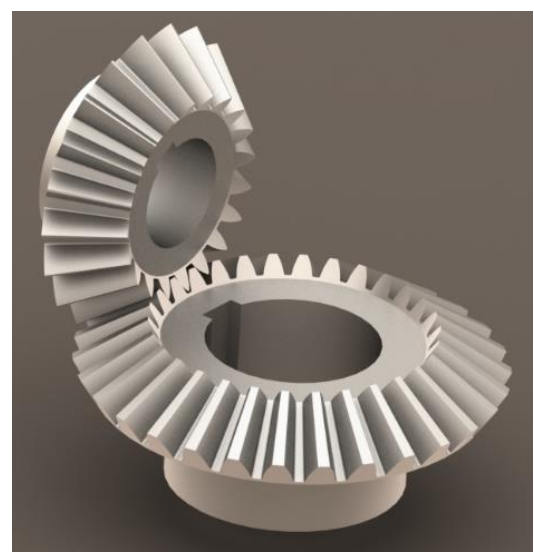

f) CAD models of the elements

Figure 3. Geometric design of a bevel gear pair having straight teeth [2] 


\section{The possibilities of the GearTeq software}

A mechanical engineer user has to know the necessary gear theorem and the mathematical and manufacturing skills which can be found in the references [4 - 11] to design connection correct gear pairs. The following gear pairs can be designed among others:

- cylindrical gear drives having straight, helical and spiroid tooth direction,

- bevel gear pairs having straight, circular, spiral tooth direction,

- cylindrical worm gear drives with different worm profiles,

- chain transmissions,

- spline fittings,

- V-belt drives,

- internal gear drives,

- planetary gear drives,

- elliptical gear drives,

- gear pairs having bypass axes.

The software can work based on the theorem of double wrapping [4,6]. It means knowing of the tooth surface of the pinion, the kinematical relations and motion parameters between the pinion and the gear it can determine the tooth surface of the gear by numerical way $[4,6]$.

The development of the software opened a big choice in the field of tooth design, rapid and connection correct CAD modelling.

Beat examinations and tooth connection analysis can be done on the saved CAD models in the SolidWorks software. There models are usable for different TCA researches.

\subsection{The application of the GearTeq software for gear design}

We designed some samples by the software where TCA could be done after the geometric design. Based on the geometric parameters and the material selection these gears are advantageously usable for the vehicle industry.

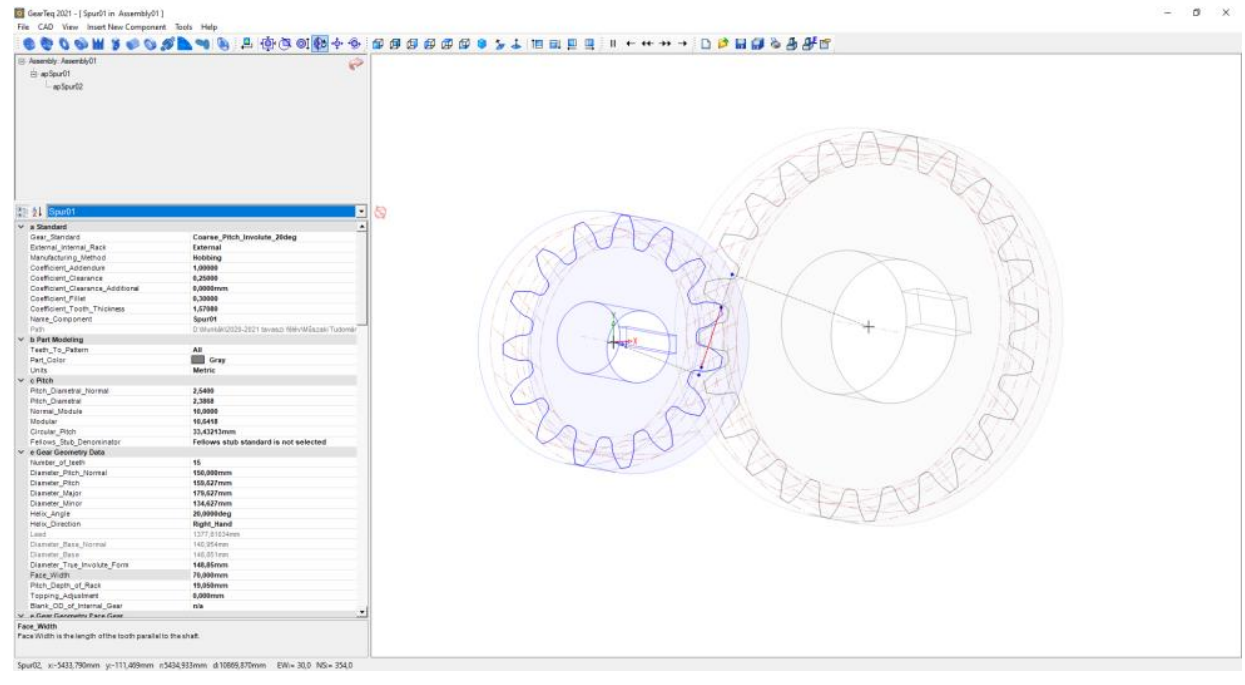

a) Design of a helical gear by GearTeq 
International Journal of Engineering and Management Sciences (IJEMS) Vol. 6. (2021). No. 2

DOI: 10.21791/IJEMS.2021.2.17.

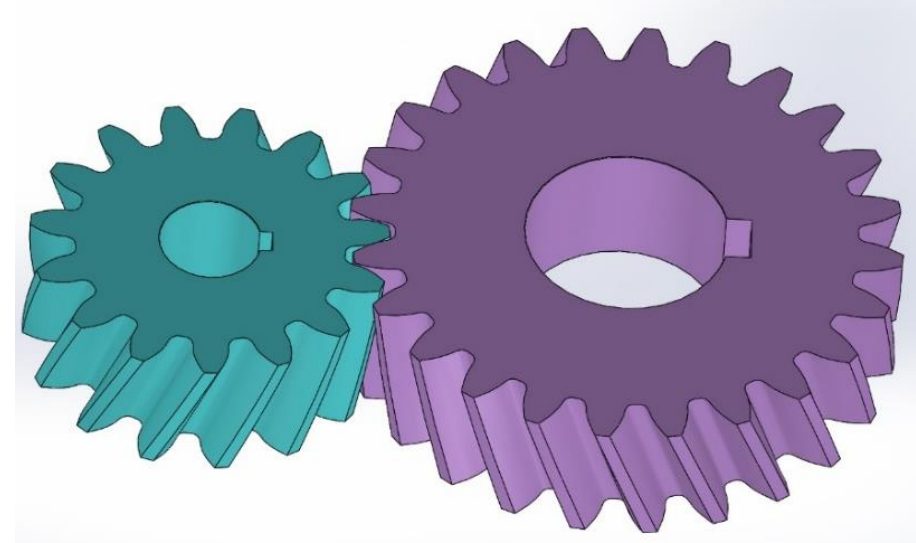

b) The CAD models of the elements 
International Journal of Engineering and Management Sciences (IJEMS) Vol. 6. (2021). No. 2

DOI: 10.21791/IJEMS.2021.2.17.

\begin{tabular}{|c|c|c|c|}
\hline SYMBOL & VALUE & UNIT & TERM \\
\hline & Coarse_Pitch_Involute_20deg & & Standard \\
\hline Pdn & 2,54 & & Normal Diametral Pitch \\
\hline \multirow[t]{2}{*}{$\mathrm{Pd}$} & 2,386819 & & Diametral Pitch \\
\hline & 10 & & Normal Modular Pitch \\
\hline $\mathrm{m}$ & 10,641778 & & Modular Pitch \\
\hline$\varnothing n$ & 20 & deg & Normal Pressure Angle \\
\hline \multirow[t]{6}{*}{$\varnothing$} & 21,173 & $\operatorname{deg}$ & Pressure Angle \\
\hline & 20 & deg & Helix Angle \\
\hline & & & \\
\hline & Gear Data & & Spur01 \\
\hline & Right_Hand & & Hand of Helix \\
\hline & 1377,8103 & $\mathrm{~mm}$ & Lead \\
\hline $\mathrm{Np}$ & 15 & & Number of Teeth \\
\hline $\mathrm{Dp}$ & 159,627 & $\mathrm{~mm}$ & Pitch Diameter \\
\hline Dpn & 150 & $\mathrm{~mm}$ & Pitch Diameter, Normal \\
\hline do & 179,627 & $\mathrm{~mm}$ & Major Diameter \\
\hline $\mathrm{dr}$ & 134,627 & $\mathrm{~mm}$ & Minor Diameter \\
\hline $\mathrm{a}$ & 10 & $\mathrm{~mm}$ & Addendum \\
\hline $\mathrm{b}$ & 12,5 & $\mathrm{~mm}$ & Dedendum \\
\hline \multirow[t]{2}{*}{$\mathrm{x}$} & 0 & & Addendum Modification Coefficient \\
\hline & & $\mathrm{mm}$ & Addendum Modification \\
\hline$d b$ & 148,851 & $\mathrm{~mm}$ & Base Diameter \\
\hline$d b n$ & 140,954 & $\mathrm{~mm}$ & Base Diameter, Normal \\
\hline TIF & 148,854 & $\mathrm{~mm}$ & True Involute Form Diameter \\
\hline ht & 22,5 & $\mathrm{~mm}$ & Whole Depth \\
\hline$p$ & 33,432 & $\mathrm{~mm}$ & Circular Pitch \\
\hline \multirow[t]{2}{*}{$\mathrm{pn}$} & 31,416 & $\mathrm{~mm}$ & Circular Pitch, Normal \\
\hline & & $\mathrm{mm}$ & Fillet Radius \\
\hline$B$ & 0,5 & $\mathrm{~mm}$ & Backlash \\
\hline $\mathrm{t}$ & 16,2161 & $\mathrm{~mm}$ & Tooth Thickness \\
\hline $\mathrm{tn}$ & 15,2381 & $\mathrm{~mm}$ & Tooth Thickness, Normal \\
\hline t & 14,8611 & $\mathrm{~mm}$ & Tooth Thickness Minimum \\
\hline \multirow[t]{7}{*}{$F$} & 70 & $\mathrm{~mm}$ & Face Width \\
\hline & & & Chordal Tooth Thickness \\
\hline & 15,741 & & Chordal Tooth Height \\
\hline & 148,854 & & Chordal Tooth Reference Circle \\
\hline & 16,659 & & Chordal Tooth Thickness \\
\hline & 16,3099 & & Chordal Tooth Thickness Minimum \\
\hline & & & Size Over Pins \\
\hline $\mathrm{dw}$ & 16,256 & $\mathrm{~mm}$ & Pin Diameter \\
\hline \multirow[t]{3}{*}{$\mathrm{M}$} & 177,906 & $\mathrm{~mm}$ & Measurement Over Pins \\
\hline & 176,965 & $\mathrm{~mm}$ & Measurement Over Pins-Minimum \\
\hline & & & Span Over Teeth \\
\hline \multirow[t]{18}{*}{$\mathrm{k}$} & 2 & & Number of Teeth to Span Over \\
\hline & 46,349 & $\mathrm{~mm}$ & Span Measurement \\
\hline & 45,972 & $\mathrm{~mm}$ & Span Measurement Minimum \\
\hline & & & Master Gear Test \\
\hline & 0 & & Master Pitch Diameter \\
\hline & & $\mathrm{mm}$ & Test Radius (Max. Act.) \\
\hline & & $\mathrm{mm}$ & Test Radius (Min. Act.) \\
\hline & AGMA-Q7 & & AGMA Quality Class \\
\hline & 0,2032 & $\mathrm{~mm}$ & Max Runout \\
\hline & 0,04318 & $\mathrm{~mm}$ & Pitch Variation \\
\hline & 0,06858 & $\mathrm{~mm}$ & Profile Tolerance \\
\hline & & $\mathrm{mm}$ & Tooth Alignment Tolerance \\
\hline & 0,08128 & $\mathrm{~mm}$ & Tooth to Tooth Composite Tolerance \\
\hline & 0,3048 & $\mathrm{~mm}$ & Total Composite Tolerance \\
\hline & 0,377 & $\mathrm{~mm}$ & Tooth Thickness Tolerance \\
\hline & & $\mathrm{mm}$ & Hob Protuberance \\
\hline & 38,7 & deg & Roll Angle at Major Diameter \\
\hline & 0,37 & deg & Roll Angle at TIF Diameter \\
\hline
\end{tabular}

\section{c) The calculated geometric parameters}

Figure 4. Design of a helical gear drive

$\left(m_{a x}=10 \mathrm{~mm}, \beta_{0}=20^{\circ}\right)$

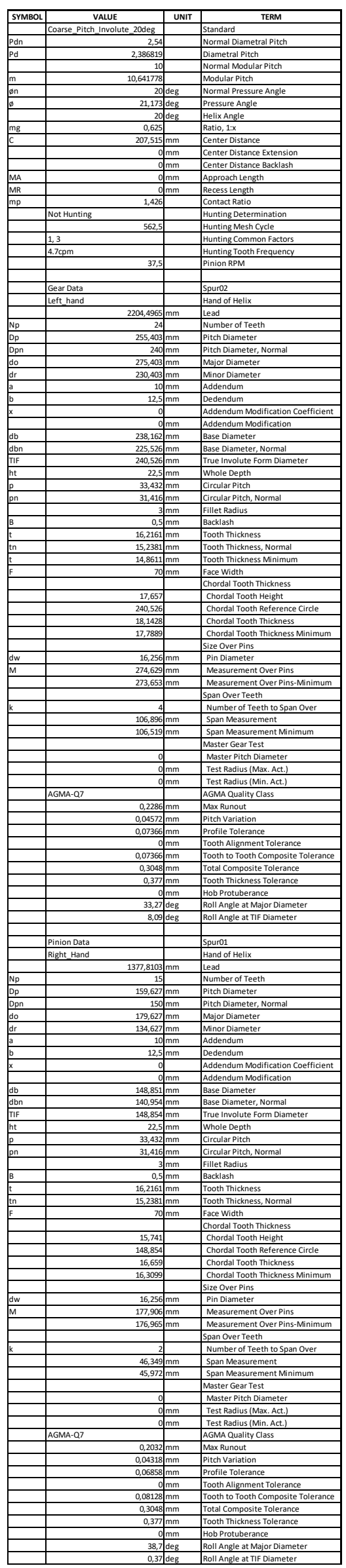




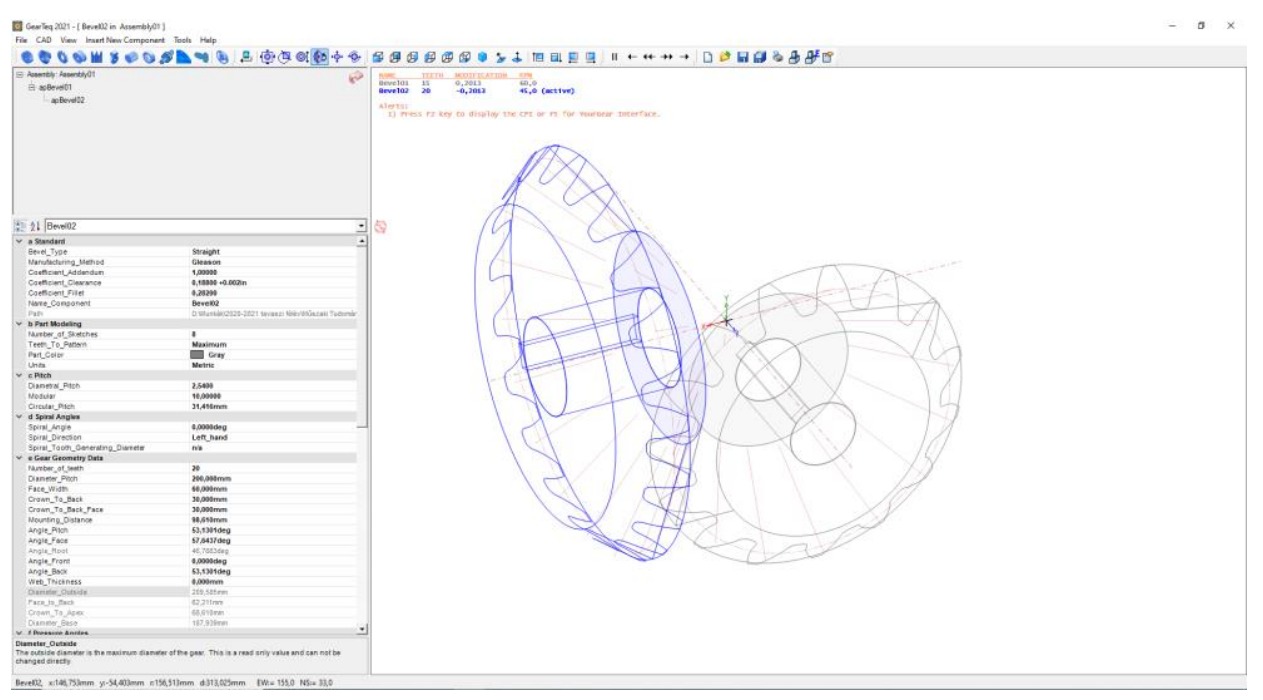

a) Design of a bevel gear having straight tooth by GearTeq

\begin{tabular}{|c|c|c|c|c|c|c|c|}
\hline SYMBOL & VALUE & UNIT & TERM & SYMBOL & VALUE & UNIT & TERM \\
\hline $\mathrm{Pd}$ & 2,54 & & Diametral Pitch & $\mathrm{Pd}$ & 2,54 & & Diametral Pitch \\
\hline$m$ & 10 & & Modular & $\mathrm{m}$ & 10 & & Modular \\
\hline \multirow[t]{3}{*}{248} & 20 & deg & Pressure Angle & 248 & 20 & deg & Pressure Angle \\
\hline & 0 & deg & Spiral Angle & & 0 & deg & Spiral Angle \\
\hline & Right_Hand & & Spiral direction & $\mathrm{mg}$ & 1,333 & & Ratio, 1:x \\
\hline$n$ & 15 & & Number of Teeth & 248 & 90 & $\operatorname{deg}$ & Shaft Angle \\
\hline $\mathrm{F}$ & 60 & $\mathrm{~mm}$ & Face Width & & Left_hand & & Spiral direction \\
\hline hk & 20 & $\mathrm{~mm}$ & Working Depth & $n$ & 20 & & Number of Teeth \\
\hline ht & 21,931 & $\mathrm{~mm}$ & Whole Depth & $\mathrm{F}$ & 60 & $\mathrm{~mm}$ & Face Width \\
\hline \multirow[t]{2}{*}{$\mathrm{Dp}$} & 150 & $\mathrm{~mm}$ & Pitch Diameter & hk & 20 & $\mathrm{~mm}$ & Working Depth \\
\hline & 36,87 & $\operatorname{deg}$ & Pitch Angle & ht & 21,931 & $\mathrm{~mm}$ & Whole Depth \\
\hline $\mathrm{AO}$ & 125 & $\mathrm{~mm}$ & \begin{tabular}{|l} 
Cone Distance \\
Cone Angle
\end{tabular} & Dp & 200 & $\mathrm{~mm}$ & Pitch Diameter \\
\hline $\mathrm{p}$ & 31,416 & $\mathrm{~mm}$ & Cone Distance & & 53,13 & deg & Pitch Angle \\
\hline $\mathrm{aP}$ & & & & AO & 125 & $\mathrm{~mm}$ & Cone Distance \\
\hline $\mathrm{bP}$ & 12,012 & $\mathrm{~mm}$ & Addendum & $\mathrm{p}$ & 31,416 & $\mathrm{~mm}$ & Circular Pitch \\
\hline \multirow[t]{4}{*}{ c } & 9,867 & $\mathrm{~mm}$ & Dedendum & $\mathrm{aP}$ & 7,987 & $\mathrm{~mm}$ & Addendum \\
\hline & 1,88 & $\mathrm{~mm}$ & Clearance & $\mathrm{bP}$ & 13,892 & $\mathrm{~mm}$ & Dedendum \\
\hline & 4,514 & deg & Dedendum Angle & |c & 1,88 & $\mathrm{~mm}$ & Clearance \\
\hline & 43,212 & deg & Face Angle of Blank & & 6,342 & deg & Dedendum Angle \\
\hline do & 32,356 & deg & Root Angle & & 57,644 & deg & Face Angle of Blank \\
\hline$\frac{\mathrm{do}}{\mathrm{xo}}$ & 169,22 & $\mathrm{~mm}$ & Outside Diameter & & 46,788 & deg & Root Angle \\
\hline$\frac{x_{0}}{t}$ & 92,792 & $\mathrm{~mm}$ & Pitch Apex To Crown & do & 209,585 & $\mathrm{~mm}$ & Outside Diameter \\
\hline \multirow[t]{13}{*}{$B$} & 16,672 & $\mathrm{~mm}$ & Tooth Thickness & xo & 68,61 & $\mathrm{~mm}$ & Pitch Apex To Crown \\
\hline & 0,5 & $\mathrm{~mm}$ & Backlash & $t$ & 13,744 & $\mathrm{~mm}$ & Tooth Thickness \\
\hline & 1 & & Addendum Coefficient & B & 0,5 & $\mathrm{~mm}$ & Backlash \\
\hline & 2,012 & $\mathrm{~mm}$ & Addendum Modification & & 1 & & Addendum Coefficient \\
\hline & 0,2013 & & Addendum Modification Coefficient & & \begin{tabular}{r|}
$-2,012$ \\
$-0,2013$
\end{tabular} & $\mathrm{~mm}$ & Addendum Modification \\
\hline & 2,82 & $\mathrm{~mm}$ & Fillet Radius & & $\frac{-0,2013}{2,82}$ & & Addendum Modification Coefficient \\
\hline & none & & AGMA Quality Class & & none & $\mathrm{mm}$ & Fillet Radius \\
\hline & $\mathrm{N} / \mathrm{A}$ & & Hunting Determination & & Not Hunting & & \\
\hline & N/A & & Hunting Mesh Cycle & & & & Hunting Determination \\
\hline & 1 & & Hunting Common Factors & & 675 & & Hunting Mesh Cycle \\
\hline & $0.0 \mathrm{cpm}$ & & Hunting Tooth Frequency & & 1,5 & & Hunting Common Factors \\
\hline & 60 & & Pinion RPM & & $11.3 \mathrm{cpm}$ & & Hunting Tooth Frequency \\
\hline & & & & & 45 & & Pinion RPM \\
\hline
\end{tabular}

b) The calculated geometric parameters 


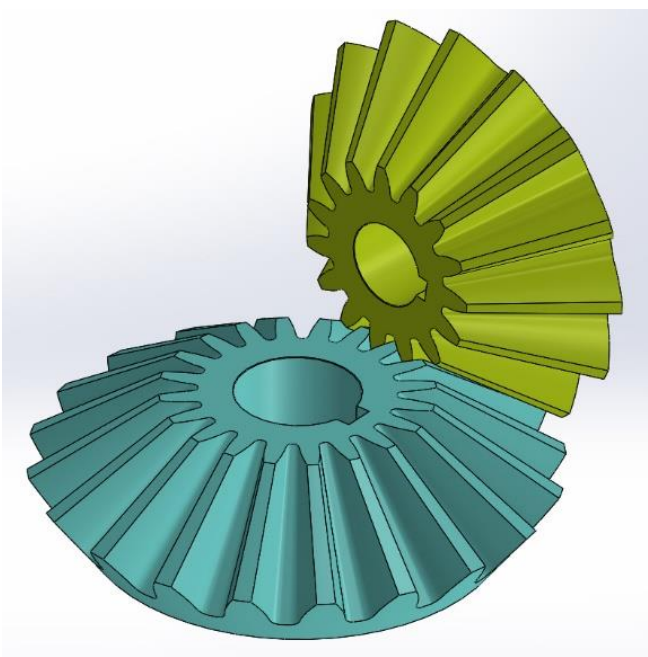

c) The CAD models of the elements

Figure 5. Design of a bevel gear having straight tooth ( $\left.m_{a x}=10 \mathrm{~mm}\right)$

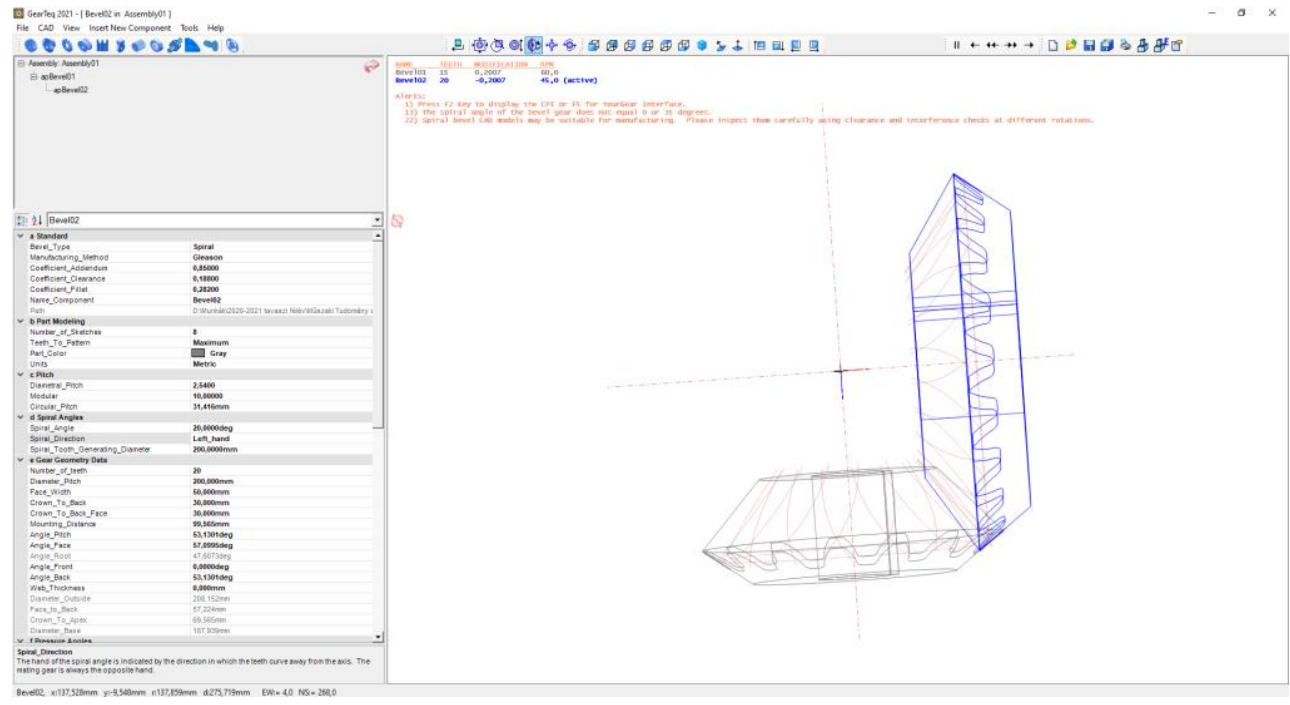

a) Design of a spiral bevel gear by GearTeq

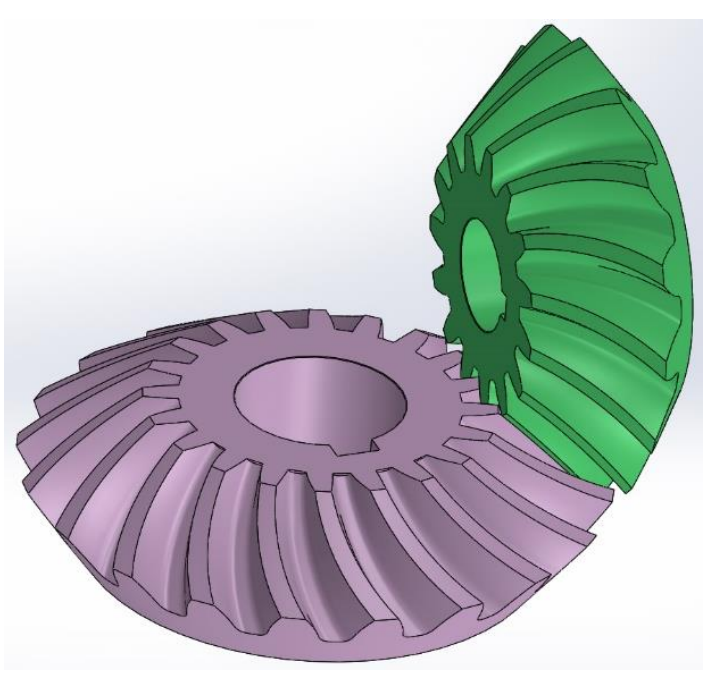

b) The CAD models of the elements 


\begin{tabular}{|c|c|c|c|}
\hline SYMBOL & VALUE & UNIT & TERM \\
\hline $\mathrm{Pd}$ & 2,54 & & Diametral Pitch \\
\hline $\mathrm{m}$ & 10 & & Modular \\
\hline \multirow[t]{3}{*}{248} & 20 & deg & Pressure Angle \\
\hline & 20 & deg & Spiral Angle \\
\hline & Right_Hand & & Spiral direction \\
\hline$n$ & 15 & & Number of Teeth \\
\hline $\mathrm{F}$ & 50 & $\mathrm{~mm}$ & Face Width \\
\hline hk & 17 & $\mathrm{~mm}$ & Working Depth \\
\hline ht & 18,88 & $\mathrm{~mm}$ & Whole Depth \\
\hline \multirow[t]{2}{*}{$\mathrm{Dp}$} & 150 & $\mathrm{~mm}$ & Pitch Diameter \\
\hline & 36,87 & deg & Pitch Angle \\
\hline $\mathrm{AO}$ & 125 & $\mathrm{~mm}$ & Cone Distance \\
\hline$p$ & 31,416 & $\mathrm{~mm}$ & Circular Pitch \\
\hline$\overline{\mathrm{aP}}$ & 10,206 & $\mathrm{~mm}$ & Addendum \\
\hline $\mathrm{bP}$ & 8,674 & $\mathrm{~mm}$ & Dedendum \\
\hline \multirow[t]{4}{*}{ c } & 1,88 & $\mathrm{~mm}$ & Clearance \\
\hline & 3,969 & deg & Dedendum Angle \\
\hline & 42,393 & deg & Face Angle of Blank \\
\hline & 32,901 & deg & Root Angle \\
\hline do & 166,33 & $\mathrm{~mm}$ & Outside Diameter \\
\hline xo & 93,876 & $\mathrm{~mm}$ & Pitch Apex To Crown \\
\hline$t$ & 16,187 & $\mathrm{~mm}$ & Tooth Thickness \\
\hline \multirow[t]{12}{*}{$B$} & 0,5 & $\mathrm{~mm}$ & Backlash \\
\hline & 0,85 & & Addendum Coefficient \\
\hline & 2,007 & $\mathrm{~mm}$ & Addendum Modification \\
\hline & 0,2007 & & Addendum Modification Coefficient \\
\hline & 2,82 & $\mathrm{~mm}$ & Fillet Radius \\
\hline & none & & AGMA Quality Class \\
\hline & $\mathrm{N} / \mathrm{A}$ & & Hunting Determination \\
\hline & $\mathrm{N} / \mathrm{A}$ & & Hunting Mesh Cycle \\
\hline & 1 & & Hunting Common Factors \\
\hline & $0.0 \mathrm{cpm}$ & & Hunting Tooth Frequency \\
\hline & 60 & & Pinion RPM \\
\hline & & & \\
\hline
\end{tabular}

\begin{tabular}{|c|c|c|c|}
\hline SYMBOL & VALUE & UNIT & TERM \\
\hline $\mathrm{Pd}$ & 2,54 & & Diametral Pitch \\
\hline $\mathrm{m}$ & 10 & & Modular \\
\hline \multirow[t]{2}{*}{248} & 20 & deg & Pressure Angle \\
\hline & 20 & deg & Spiral Angle \\
\hline $\mathrm{mg}$ & 1,333 & & Ratio, 1:x \\
\hline \multirow[t]{2}{*}{248} & 90 & deg & Shaft Angle \\
\hline & Left_hand & & Spiral direction \\
\hline$n$ & 20 & & Number of Teeth \\
\hline $\mathrm{F}$ & 50 & $\mathrm{~mm}$ & Face Width \\
\hline hk & 17 & $\mathrm{~mm}$ & Working Depth \\
\hline ht & 18,88 & $\mathrm{~mm}$ & Whole Depth \\
\hline \multirow[t]{2}{*}{$D p$} & 200 & $\mathrm{~mm}$ & Pitch Diameter \\
\hline & 53,13 & deg & Pitch Angle \\
\hline $\mathrm{AO}$ & 125 & $\mathrm{~mm}$ & Cone Distance \\
\hline$p$ & 31,416 & $\mathrm{~mm}$ & Circular Pitch \\
\hline $\mathrm{aP}$ & 6,794 & $\mathrm{~mm}$ & Addendum \\
\hline $\mathrm{bP}$ & 12,086 & $\mathrm{~mm}$ & Dedendum \\
\hline \multirow[t]{4}{*}{ c } & 1,88 & $\mathrm{~mm}$ & Clearance \\
\hline & 5,523 & deg & Dedendum Angle \\
\hline & 57,099 & deg & Face Angle of Blank \\
\hline & 47,607 & deg & Root Angle \\
\hline do & 208,152 & $\mathrm{~mm}$ & Outside Diameter \\
\hline xo & 69,565 & $\mathrm{~mm}$ & Pitch Apex To Crown \\
\hline $\mathrm{t}$ & 14,229 & $\mathrm{~mm}$ & Tooth Thickness \\
\hline \multirow[t]{12}{*}{ B } & 0,5 & $\mathrm{~mm}$ & Backlash \\
\hline & 0,85 & & Addendum Coefficient \\
\hline & $-2,007$ & $\mathrm{~mm}$ & Addendum Modification \\
\hline & $-0,2007$ & & Addendum Modification Coefficient \\
\hline & 2,82 & $\mathrm{~mm}$ & Fillet Radius \\
\hline & none & & AGMA Quality Class \\
\hline & Not Hunting & & Hunting Determination \\
\hline & 675 & & Hunting Mesh Cycle \\
\hline & 1,5 & & Hunting Common Factors \\
\hline & $11.3 \mathrm{cpm}$ & & Hunting Tooth Frequency \\
\hline & 45 & & Pinion RPM \\
\hline & & & \\
\hline
\end{tabular}

c) The calculated geometric parameters

Figure 6. Design of a spiral bevel gear $\left(m_{a x}=10 \mathrm{~mm}\right)$

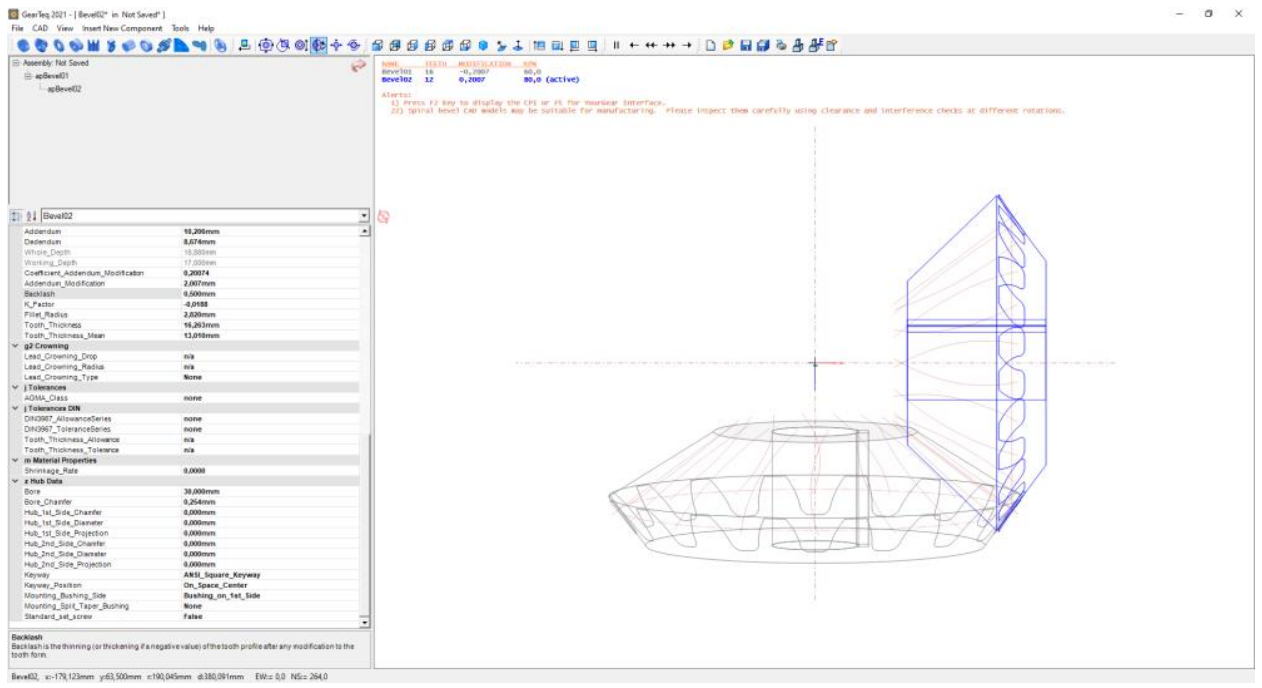

a) Design of a Zerol bevel gear by GearTeq 


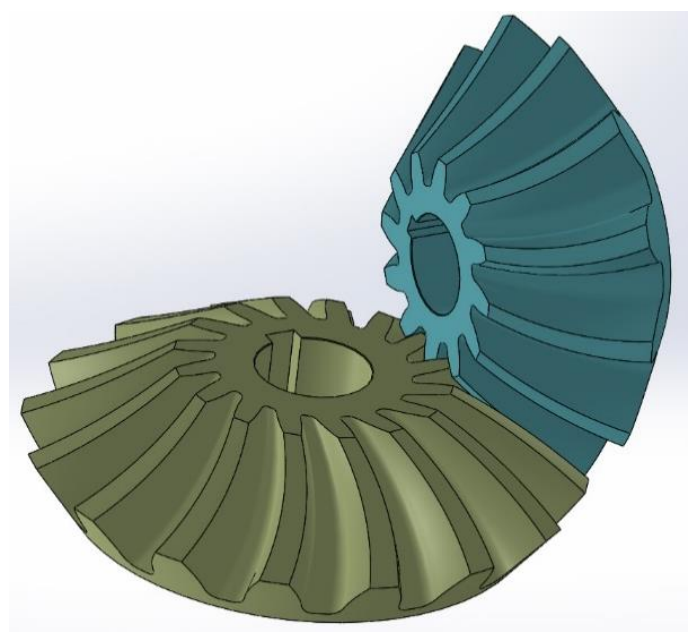

b) The CAD models of the elements

\begin{tabular}{|c|c|c|c|}
\hline SYMBOL & VALUE & UNIT & TERM \\
\hline $\mathrm{Pd}$ & 2,54 & & Diametral Pitch \\
\hline $\mathrm{m}$ & 10 & & Modular \\
\hline 248 & 20 & deg & Pressure Angle \\
\hline & 0 & deg & Spiral Angle \\
\hline & Right_Hand & & Spiral direction \\
\hline$n$ & 16 & & Number of Teeth \\
\hline $\mathrm{F}$ & 50 & $\mathrm{~mm}$ & Face Width \\
\hline hk & 17 & $\mathrm{~mm}$ & Working Depth \\
\hline ht & 18,88 & $\mathrm{~mm}$ & Whole Depth \\
\hline \multirow[t]{2}{*}{$\mathrm{Dp}$} & 160 & $\mathrm{~mm}$ & Pitch Diameter \\
\hline & 53,13 & deg & Pitch Angle \\
\hline $\mathrm{AO}$ & 100 & $\mathrm{~mm}$ & Cone Distance \\
\hline $\mathrm{p}$ & 31,416 & $\mathrm{~mm}$ & Circular Pitch \\
\hline $\mathrm{aP}$ & 6,794 & $\mathrm{~mm}$ & Addendum \\
\hline bP & 12,086 & $\mathrm{~mm}$ & Dedendum \\
\hline \multirow[t]{4}{*}{ c } & 1,88 & $\mathrm{~mm}$ & Clearance \\
\hline & 6,891 & deg & Dedendum Angle \\
\hline & 58,087 & deg & Face Angle of Blank \\
\hline & 46,239 & deg & Root Angle \\
\hline do & 168,152 & $\mathrm{~mm}$ & Outside Diameter \\
\hline$x o$ & 54,565 & $\mathrm{~mm}$ & Pitch Apex To Crown \\
\hline $\mathrm{t}$ & 14,153 & $\mathrm{~mm}$ & Tooth Thickness \\
\hline \multirow[t]{12}{*}{$B$} & 0,5 & $\mathrm{~mm}$ & Backlash \\
\hline & 0,85 & & Addendum Coefficient \\
\hline & $-2,007$ & $\mathrm{~mm}$ & Addendum Modification \\
\hline & $-0,2007$ & & Addendum Modification Coefficient \\
\hline & 2,82 & $\mathrm{~mm}$ & Fillet Radius \\
\hline & none & & AGMA Quality Class \\
\hline & $\mathrm{N} / \mathrm{A}$ & & Hunting Determination \\
\hline & $\mathrm{N} / \mathrm{A}$ & & Hunting Mesh Cycle \\
\hline & 1 & & Hunting Common Factors \\
\hline & $0.0 \mathrm{cpm}$ & & Hunting Tooth Frequency \\
\hline & 60 & & Pinion RPM \\
\hline & & & \\
\hline
\end{tabular}

\begin{tabular}{|c|c|c|c|}
\hline SYMBOL & VALUE & UNIT & TERM \\
\hline $\mathrm{Pd}$ & 2,54 & & Diametral Pitch \\
\hline $\mathrm{m}$ & 10 & & Modular \\
\hline 248 & 20 & deg & Pressure Angle \\
\hline & 0 & deg & Spiral Angle \\
\hline $\mathrm{mg}$ & 0,75 & & Ratio, 1:x \\
\hline 248 & 90 & deg & Shaft Angle \\
\hline & Left_hand & & Spiral direction \\
\hline n & 12 & & Number of Teeth \\
\hline $\mathrm{F}$ & 50 & $\mathrm{~mm}$ & Face Width \\
\hline hk & 17 & $\mathrm{~mm}$ & Working Depth \\
\hline ht & 18,88 & $\mathrm{~mm}$ & Whole Depth \\
\hline \multirow[t]{2}{*}{$\mathrm{Dp}$} & 120 & $\mathrm{~mm}$ & Pitch Diameter \\
\hline & 36,87 & deg & Pitch Angle \\
\hline $\mathrm{AO}$ & 100 & $\mathrm{~mm}$ & Cone Distance \\
\hline $\mathrm{p}$ & 31,416 & $\mathrm{~mm}$ & Circular Pitch \\
\hline aP & 10,206 & $\mathrm{~mm}$ & Addendum \\
\hline $\mathrm{bP}$ & 8,674 & $\mathrm{~mm}$ & Dedendum \\
\hline \multirow[t]{4}{*}{ c } & 1,88 & $\mathrm{~mm}$ & Clearance \\
\hline & 4,957 & deg & Dedendum Angle \\
\hline & 43,761 & deg & Face Angle of Blank \\
\hline & 31,913 & deg & Root Angle \\
\hline do & 136,33 & $\mathrm{~mm}$ & Outside Diameter \\
\hline xo & 73,876 & $\mathrm{~mm}$ & Pitch Apex To Crown \\
\hline $\mathrm{t}$ & 16,263 & $\mathrm{~mm}$ & Tooth Thickness \\
\hline \multirow[t]{12}{*}{ B } & 0,5 & $\mathrm{~mm}$ & Backlash \\
\hline & 0,85 & & Addendum Coefficient \\
\hline & 2,007 & $\mathrm{~mm}$ & Addendum Modification \\
\hline & 0,2007 & & Addendum Modification Coefficient \\
\hline & 2,82 & $\mathrm{~mm}$ & Fillet Radius \\
\hline & none & & AGMA Quality Class \\
\hline & Not Hunting & & Hunting Determination \\
\hline & 960 & & Hunting Mesh Cycle \\
\hline & $1,2,4$ & & Hunting Common Factors \\
\hline & $20.0 \mathrm{cpm}$ & & Hunting Tooth Frequency \\
\hline & 80 & & Pinion RPM \\
\hline & & & \\
\hline
\end{tabular}

c) The calculated geometric parameters

Figure 7. Design of a Zerol bevel gear ( $\left.m_{a x}=10 \mathrm{~mm}\right)$ 
International Journal of Engineering and Management Sciences (IJEMS) Vol. 6. (2021). No. 2

DOI: 10.21791/IJEMS.2021.2.17.

\begin{tabular}{|c|c|c|c|}
\hline SYMBOL & VALUE & |UNIT & TERM \\
\hline & Coarse_Pitch_Involute_20deg & & Standard \\
\hline Pdn & 2,54 & & Normal Diametral Pitch \\
\hline \multirow[t]{2}{*}{$\mathrm{Pd}$} & 2,453452 & & Diametral Pitch \\
\hline & 10 & & Normal Modular Pitch \\
\hline $\mathrm{m}$ & 10,352762 & & Modular Pitch \\
\hline$\emptyset \mathrm{n}$ & & deg & Normal Pressure Angle \\
\hline \multirow[t]{6}{*}{$\phi$} & 20,647 & 7 deg & Pressure Angle \\
\hline & 15 & 5 deg & Helix Angle \\
\hline & & & \\
\hline & Gear Data & & Spur01 \\
\hline & Left_hand & & Hand of Helix \\
\hline & 2427,6363 & $\mathrm{~mm}$ & Lead \\
\hline $\mathrm{Np}$ & 20 & & Number of Teeth \\
\hline $\mathrm{Dp}$ & 207,055 & $5 \mathrm{~mm}$ & Pitch Diameter \\
\hline $\mathrm{Dpn}$ & 200 & $\mathrm{~mm}$ & Pitch Diameter, Normal \\
\hline do & 227,055 & $\mathrm{~mm}$ & Major Diameter \\
\hline $\mathrm{dr}$ & 182,055 & $5 \mathrm{~mm}$ & Minor Diameter \\
\hline $\mathrm{a}$ & & $\mathrm{mm}$ & Addendum \\
\hline $\mathrm{b}$ & 12,5 & $\mathrm{~mm}$ & Dedendum \\
\hline \multirow[t]{2}{*}{$x$} & & o & Addendum Modification Coefficient \\
\hline & & $\mathrm{mm}$ & Addendum Modification \\
\hline$d b$ & 193,756 & $5 \mathrm{~mm}$ & Base Diameter \\
\hline$d b n$ & 187,939 & $\mathrm{~mm}$ & Base Diameter, Normal \\
\hline TIF & 194,2 & $\mathrm{~mm}$ & True Involute Form Diameter \\
\hline ht & 22,5 & $5 \mathrm{~mm}$ & Whole Depth \\
\hline $\mathrm{p}$ & 32,524 & $4 \mathrm{~mm}$ & Circular Pitch \\
\hline \multirow[t]{2}{*}{$\mathrm{pn}$} & 31,416 & $\mathrm{~mm}$ & Circular Pitch, Normal \\
\hline & & $\mathrm{mm}$ & Fillet Radius \\
\hline$B$ & 0,5 & $\mathrm{~mm}$ & Backlash \\
\hline $\mathrm{t}$ & 15,7621 & $\mathrm{~mm}$ & Tooth Thickness \\
\hline tn & 15,225 & $5 \mathrm{~mm}$ & Tooth Thickness, Normal \\
\hline t & 14,848 & $\mathrm{~mm}$ & Tooth Thickness Minimum \\
\hline \multirow[t]{7}{*}{$\mathrm{F}$} & 60 & $\mathrm{~mm}$ & Face Width \\
\hline & & & Chordal Tooth Thickness \\
\hline & 10,196 & & \begin{tabular}{|l|} 
Chordal Tooth Height \\
\end{tabular} \\
\hline & 207,185 & & Chordal Tooth Reference Circle \\
\hline & 15,1725 & & Chordal Tooth Thickness \\
\hline & 14,7963 & & Chordal Tooth Thickness Minimum \\
\hline & & & Size Over Pins \\
\hline$d w$ & 16,256 & $6 \mathrm{~mm}$ & Pin Diameter \\
\hline \multirow[t]{3}{*}{ M } & 226,219 & $9 \mathrm{~mm}$ & Measurement Over Pins \\
\hline & 225,258 & $\mathrm{~mm}$ & Measurement Over Pins-Minimum \\
\hline & & & Span Over Teeth \\
\hline \multirow[t]{18}{*}{$\mathrm{k}$} & 0 & & Number of Teeth to Span Over \\
\hline & $-12,122$ & $2 \mathrm{~mm}$ & Span Measurement \\
\hline & $-12,499$ & $9 \mathrm{~mm}$ & Span Measurement Minimum \\
\hline & & & Master Gear Test \\
\hline & $\underline{0}$ & & Master Pitch Diameter \\
\hline & & $\mathrm{mm}$ & Test Radius (Max. Act.) \\
\hline & & $\mathrm{mm}$ & $\begin{array}{l}\text { Test Radius (Min. Act.) } \\
\end{array}$ \\
\hline & AGMA-Q7 & & AGMA Quality Class \\
\hline & 0,2159 & $9 \mathrm{~mm}$ & Max Runout \\
\hline & 0,04572 & $\mathrm{~mm}$ & Pitch Variation \\
\hline & 0,07112 & $2 \mathrm{~mm}$ & Profile Tolerance \\
\hline & & $\mathrm{mm}$ & Tooth Alignment Tolerance \\
\hline & 0,07366 & $6 \mathrm{~mm}$ & Tooth to Tooth Composite Tolerance \\
\hline & 0,2794 & $4 \mathrm{~mm}$ & Total Composite Tolerance \\
\hline & 0,377 & $\mathrm{~mm}$ & Tooth Thickness Tolerance \\
\hline & & $\mathrm{mm}$ & Hob Protuberance \\
\hline & 35 & Sdeg & Roll Angle at Major Diameter \\
\hline & 3,88 & $3 \operatorname{deg}$ & Roll Angle at TIF Diameter \\
\hline
\end{tabular}

a) The calculated geometric parameters

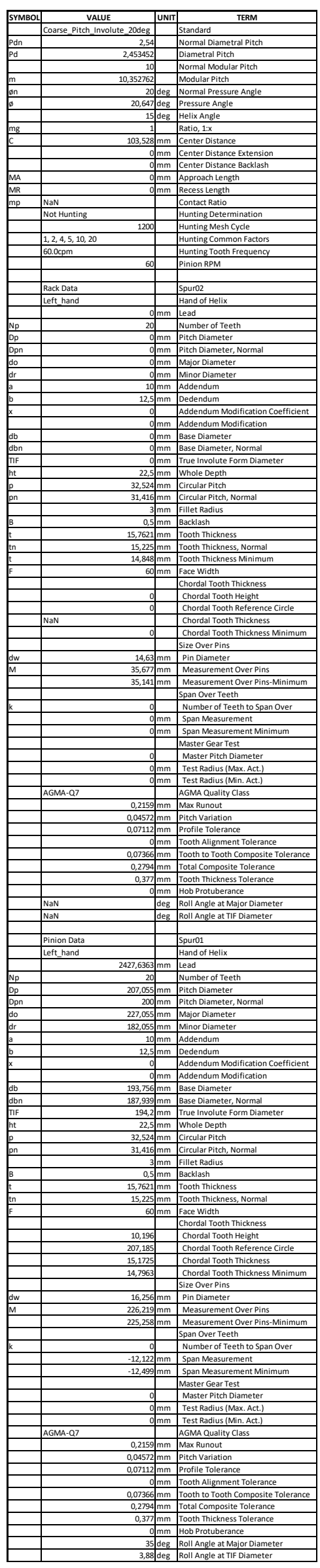




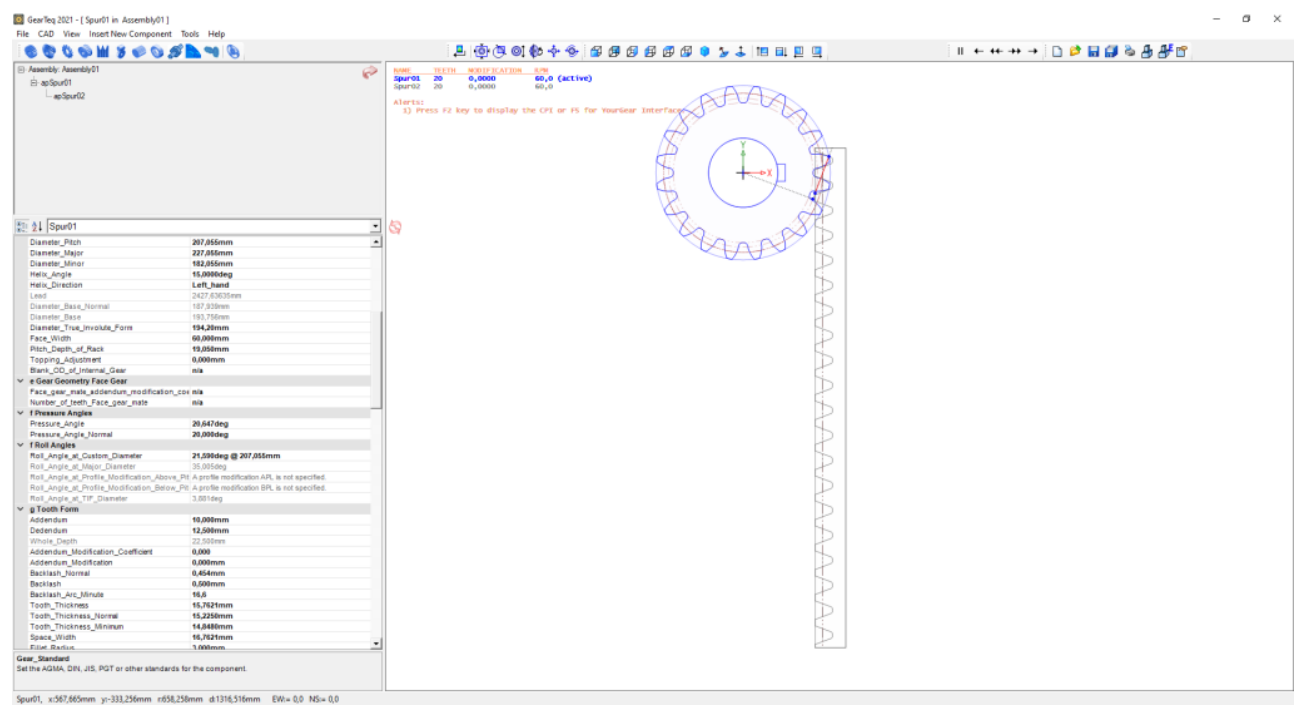

b) Design of a helical gear - gear rack connection by GearTeq

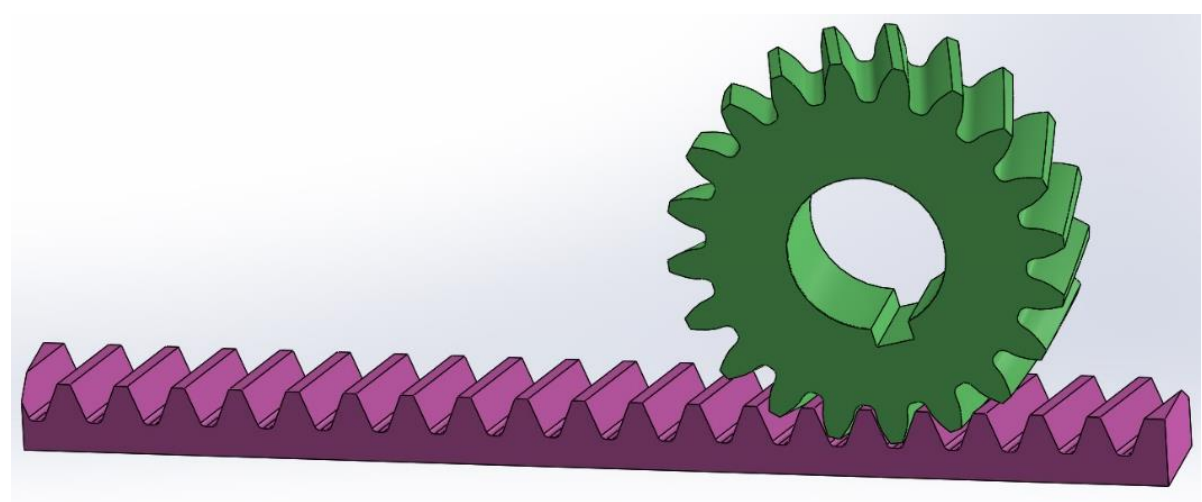

c) The CAD models of the elements

Figure 8. Design of a helical gear - gear rack connection $\left(m_{a x}=10 \mathrm{~mm}, \beta_{0}=15^{\circ}\right)$

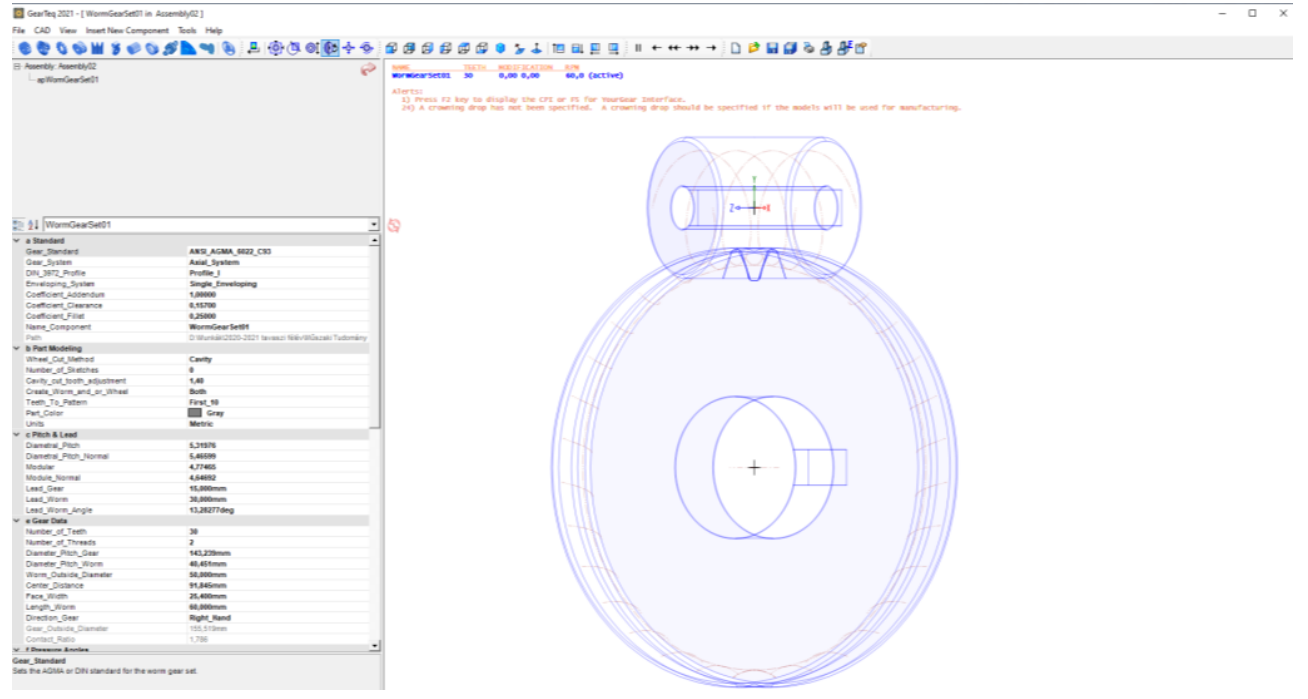

a) Design of a cylindrical worm gear drive by GearTeq 


\begin{tabular}{|c|c|c|c|}
\hline SYMBOL & VALUE & UNIT & TERM \\
\hline & ANSI AGMA 6022 C93 & & Standard \\
\hline & 5,31976 & & Diametral Pitch \\
\hline & 5,46599 & & Diametral Pitch, Normal \\
\hline & 4,77465 & $\mathrm{~mm}$ & Module \\
\hline & 4,64692 & $\mathrm{~mm}$ & Module, Normal \\
\hline & 15 & $\mathrm{~mm}$ & Lead, Wheel \\
\hline & 30 & $\mathrm{~mm}$ & Lead, Worm \\
\hline & 13,283 & deg & Lead Angle, Worm \\
\hline & 1 & & Coefficient, Addendum \\
\hline & 0,157 & & Coefficient, Clearance \\
\hline & 0,25 & & Coefficient, Fillet \\
\hline $\mathrm{N}$ & 2 & & Number of Worm Threads \\
\hline \multirow[t]{19}{*}{$\mathrm{N}$} & 30 & & Number of Teeth \\
\hline & Right Hand & & Direction of Wheel \\
\hline & 143,239 & $\mathrm{~mm}$ & Pitch Diameter, Wheel \\
\hline & 40,451 & $\mathrm{~mm}$ & Pitch Diameter, Worm \\
\hline & 50 & $\mathrm{~mm}$ & Outside Diameter, Worm \\
\hline & 91,845 & $\mathrm{~mm}$ & Center Distance \\
\hline & 25,4 & $\mathrm{~mm}$ & Face Width, Wheel \\
\hline & 60 & $\mathrm{~mm}$ & Length, Worm \\
\hline & 155,519 & $\mathrm{~mm}$ & Outside Diameter, Wheel \\
\hline & 1,786 & $\mathrm{~mm}$ & Contact Ratio \\
\hline & 20,505 & deg & Pressure Angle \\
\hline & 20 & deg & Pressure Angle, Normal \\
\hline & 0 & $\mathrm{~mm}$ & Addendum Mod. Coef., Wheel \\
\hline & 0 & $\mathrm{~mm}$ & Addendum Mod. Coef., Worm \\
\hline & 0 & $\mathrm{~mm}$ & Addendum Mod., Wheel \\
\hline & 0 & $\mathrm{~mm}$ & Addendum Mod., Worm \\
\hline & 0,1 & $\mathrm{~mm}$ & Backlash, Wheel \\
\hline & 0,097 & $\mathrm{~mm}$ & Backlash, Wheel Normal \\
\hline & 0,1 & $\mathrm{~mm}$ & Backlash, Worm \\
\hline $\mathrm{aP}$ & 4,775 & $\mathrm{~mm}$ & Addendum, Wheel \\
\hline \multirow[t]{2}{*}{$\mathrm{aP}$} & 4,775 & $\mathrm{~mm}$ & Addendum, Worm \\
\hline & 0,097 & $\mathrm{~mm}$ & Backlash, Worm Normal \\
\hline $\mathrm{bP}$ & 5,524 & $\mathrm{~mm}$ & Dedendum, Wheel \\
\hline \multirow[t]{7}{*}{$\mathrm{bP}$} & 5,524 & $\mathrm{~mm}$ & Dedendum, Worm \\
\hline & 7,4 & $\mathrm{~mm}$ & Tooth Thickness, Wheel \\
\hline & 7,4 & $\mathrm{~mm}$ & Tooth Thickness, Worm \\
\hline & 1,194 & $\mathrm{~mm}$ & Fillet Radius \\
\hline & 8,007 & $\mathrm{~mm}$ & Pin and Ball Diameter \\
\hline & 51,498 & $\mathrm{~mm}$ & Worm Measurement Over 3 Pins \\
\hline & 154,14 & $\mathrm{~mm}$ & Wheel Measurement Over 2 Balls \\
\hline
\end{tabular}

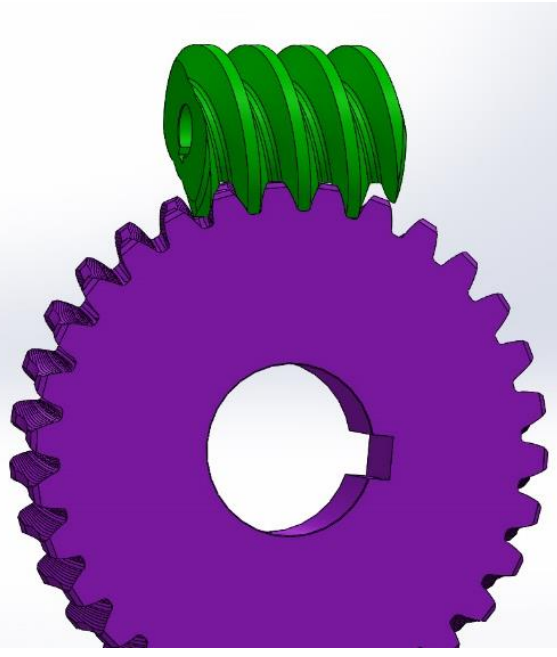

c) The CAD models of the elements

b) The calculated geometric parameters

Figure 9. Design of a cylindrical worm gear drive $\left(m_{a x}=4.77 \mathrm{~mm}\right)$

\section{Conclusion}

The aim of this study is to show the design possibilities of different types of gears in classical (unique program) and automatic ways (usage of GearTeq). Unique program is needed for the determination of the mechanical parameters including the geometric and numerical equations in case of classical way. This program can save the calculated parameters and the profile curve into a txt file. This file is readable for the SolidWorks software to build up the CAD models of the elements. On the second hand knowing the manufacturing, gear and mathematical theorem the gears can be designed by GearTeq software (automatic way). This program can tightly cooperate with the SolidWorks. After the geometric design the gear parameters can be saved into MS Excel. The CAD 
models can be imported into the SolidWorks. We showed the modelling processes in both cases and some applications. The designed gears can be usable for other researches (TCA). This overall gear design process is usable for solutions of different engineering constructions where the application of gears is needed.

\section{Acknowledgement}

Project no. TKP2020-NKA-04 has been implemented with the support provided from the National Research, Development and Innovation Fund of Hungary, financed under the 2020-4.1.1-TKP2020 funding scheme.

\section{References}

[1] Bodzás, S. (2018) 'Ferde fogazatú fogaskerékpárok számítógéppel segített tervezése és modellezése = Computer Aided Designing and Modelling of Helical Gear Pairs', In: Bodzás, Sándor; Antal, Tamás (szerk.) Múszaki Tudomány az Észak-Kelet Magyarországi Régióban 2018: konferencia előadásai Debrecen, Magyarország : Debreceni Akadémiai Bizottság Müszaki Szakbizottság (2018) 442 p. pp. 25-40. , 16 p

[2] Bodzás, S. (2020) 'Computer-aided design and loaded tooth contact analyses of bevel gear pair having straight teeth by different loaded torques', MECHANICS \& INDUSTRY $21: 1$ pp. 1-15. Paper: 109,15 p. (2020)

[3] Bodzás, S. (2020) 'Fogazott hajtópárok tervezése, modellezése és fogazatkapcsolódási (TCA) elemzése' DEBRECENI SZEMLE 28 : 4 pp. 440-453. , 14 p.

[4] Litvin, F. L., Fuentes A., A. (2004) Gear Geometry and Applied Theory, Cambridge University Press, ISBN 9780521815178

[5] Bercsey, T. (1977) 'Toroidhajtások elmélete', Candidate dissertation, Budapest

[6] Dudás, I. (2004) 'The Theory and Practice of Worm Gear Drives', Kogan Page US., USA

[7] Maros, D., Killman, V., Rohonyi, V. (1970) 'Csigahajtások', Müszaki Könyvkiadó, Budapest

[8] Goldfarb, V., Trubachev, E., Barmina, N. (2018) 'Advanced Gear Engineering', Springer, p. 197., ISBN 978-3-319-60398-8

[9] Gołębski, R., Szarek, A. (2019) Diagnosis of the Operational Gear Wheel Wear Technical Gazette, 26(3), 658-661., https://doi.org/10.17559/TV-20180321171428

[10] Radzevich, S. P. (2016). 'Dudley's Handbook of Practical Gear Design and Manufacture', Third edition, CRC Press, p. 656, ISBN 9781498753104

[11] Terplán, Z. (1975). 'Gépelemek IV.', Kézirat, Tankönyvkiadó, Budapest, p. 220. 